\title{
A Dual Percolation Model for Predicting the Connectivity of Fractured Porous Media ${ }^{1}$
}

\author{
Pingkang Wang ${ }^{a}$, Xuhui Zhang ${ }^{b *}$, Xiaobing $\mathrm{Lu}^{b}$, Wei Zheng ${ }^{b}$, and Qingjie Liu ${ }^{c}$ \\ ${ }^{a}$ Centre for Gas Hydrate, Oil and Gas Survey, China Geological Survey, Beijing, China \\ E-mail:wangpk@cags.ac.cn \\ ${ }^{b}$ Key Laboratory for Mechanics of Fluid Solid Coupling System, Institute of Mechanics, Chinese Academy of Sciences, Beijing, China \\ *E-mail: zhangxuhui@imech.ac.cn, xblu@imech.ac.cn, tulixue2010@163.com \\ ${ }^{c}$ State Key Laboratory for Enhanced Oil Recovery, Research Institute of Exploration and Development of PetroChina, Beijing, China \\ E-mail: lqj@petrochina.com.cn \\ Received September 16, 2014
}

\begin{abstract}
This paper presents a dual-percolation model coupling the percolation theory and the fracture percolation theory to study the conductivity of the fractured porous media. The Monte-Carlo method is used in the numerical simulation. First an appropriate computing scale by considering the calculation precision and elapsed time together is validated. Then, two parameters, $A_{0}$ and $D$ are presented in this model to determine the conductivity of the media. Generally the media can be blocked by itself in the condition of $D>2$. However, the increase of pore connection and the randomness of fracture direction may release the selfblockage, increase the conductivity and make the dual porous media dissipated. A few long fractures can play a great role in the connection of media.
\end{abstract}

Keywords: Dual-percolation, connectivity, fracture percolation, pore percolation

DOI: $10.1134 / \mathrm{S} 0097807816120095$

\section{INTRODUCTION}

The connectivity evaluation of reservoirs is a very important issue in many engineering practices. For example, during the geological sequestration of captured $\mathrm{CO}_{2}$, the connectivity of reservoir determines the effects of injection of $\mathrm{CO}_{2}$ and the connectivity of the cap rock determines whether the injected $\mathrm{CO}_{2}$ can be stored safely for an enough time. During oil and gas production, the connectivity of reservoir determines whether oil and gas can be exploited [1-3]. However, practical reservoirs are fractured porous media, in which the flow can pass through the pores and fractures.

Fractured reservoirs are complex, containing geological heterogeneities (i.e., fractures) on length scale from microns to kilometers. These heterogeneities have significant impact on the flow behavior and have to be modeled to make reliable prediction of reservoir performance. However, very few direct measurements are available of the flow properties, and there is a great deal of uncertainty about the spatial distribution of the fractures. The major issue in fracture hydrology is to determine the permeability and its behavior of the fracture networks [4, 5].

\footnotetext{
${ }^{1}$ The article is published in the original.
}

The conventional approach to study the impact of geological uncertainty on reservoir recovery is to build a detailed reservoir model using geophysical and geological data, and then perform flow simulation. If fractures are poorly interconnected and the matrix rock is relatively impermeable, the flow may be blocked in the network of fractures. Otherwise, if the matrix is permeable and the fractures are regular and highly interconnected, fractures and matrix can be treated as separate continuums occupying the entire domain. In order to estimate the reservoir performance parameters, it is necessary to construct reservoir models (with associated probability) and run flow simulations. This method is time consuming and computationally expensive [6]. Therefore, there is a great incentive to produce much simpler physically-based models to quickly predict uncertainty in performance.

Percolation theory is an effective method to investigate the connectivity of reservoir [7-9]. Imaging a typical reservoir model constructed with an objectbased technique, in which geometric objects representing geological entities (e.g., fractures, pores) are placed randomly in space. Then the connectivity and conductivity (i.e., effective permeability) can be estimated directly using percolation theory. This method can easily estimate the effects of complex geometry in a fraction of a second on a spreadsheet, but it ignores 


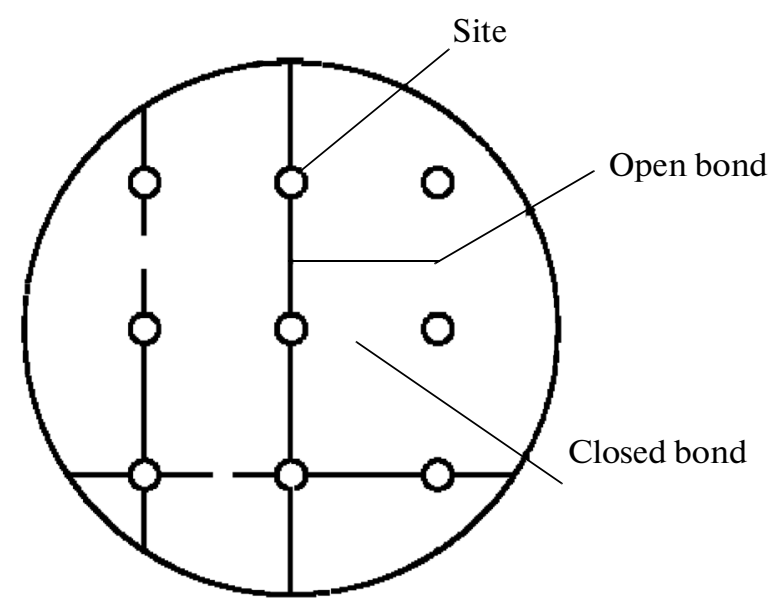

Fig. 1. Sketch of bond percolation mode in local zone.

much of the flow physics and subtleties of the heterogeneity distribution, including the effects of fracture's thickness.

A new porous and fractured percolation model is presented in this paper. This model mainly based on the characteristics of rich fractures and channels can be used to investigate the connectivity of porous and fractured dual media, and the effects of fracture length and direction can be also considered.

\section{INTRODUCTION OF PERCOLATION THEORY}

\section{Porous Percolation}

Percolation theory is a classical model of the connectivity in geometrically complex systems first developed in the late 1950s [10]. The global static and dynamic properties of such systems are linked to the density of objects (e.g., the fractures and pores in this study) placed randomly in space. The full description of this theory and its applications can be found elsewhere [11].

Some general references can be given on this topic which summarize the knowledge accumulated over the years from different points of view [12-16]. In all these studies and to the best of our knowledge, the fracture properties are assumed to be uniform and generally constant for the whole network. There are several numerical simulation studies in the literature suggesting an anomalous displacement behavior related to the fractal nature of the clusters [17-19]. However, there is little experimental evidence for the reality and practical detectability of anomalous diffusion so far [20-22].
In percolation theory a medium is defined as an infinite set of sites. A fluid flows between these sites along paths which connect certain pairs of sites (these paths are called bonds). Thus two types of percolation model are introduced here [8]: site percolation and bond percolation (Fig. 1). Normally sites lie on a regular lattice and only bonds between the nearest neighbors are considered. Site percolation involves a probability $p$ that any site is open independently of the other sites. In bond percolation the probability is for any bond to be open. A path is a sequence of sites connected by open bonds. In either case a cluster is a set of sites in which any two are connected by a path. It is found that a $p_{\mathrm{c}}$ exists so that for $p<p_{\mathrm{c}}$ only finite-size clusters exist, but for $p>p_{\mathrm{c}}$ infinite ones appear (Figs. $2 \mathrm{a}-\mathrm{c}$ ). As for bond percolation formed by quadrangle type structure, this critical probability $p_{\mathrm{c}}$ is near 0.5 (Fig. 3).

\section{Dual Percolation Model}

Dual percolation model is constituted of fracture network and matrix percolation network. Each fracture's center is mapped as a "site" of matrix porous network, and the "bond" is mapped as the connection between porosity. Fracture network is used to model the flow in fractures while matrix network is used to model the flow in porosity and defects. The coupling of these two types of models can simulate the formation of inter-porous flow channel.

In this paper the quadrangle network is used, with each fracture having four "bonds" to connect neighboring fractures (Fig. 4). The connectivity of these bonds is determined by a probability $p$ : if the probability of connection between any two bonds is larger than a critical value, these two bonds are taken as connected. Furthermore, if any two fractures are intersected, they are considered to be connected. The main point is to find the conditions required to form a connective cluster. The following assumptions are adopted in the analysis in the next section: 1-The fracture center formed by Poisson's distribution is taken as the locating point; 2 - The fracture length obeys normal distribution; 3-Fracture angles are uniformly distributed in the range of $\left[+\alpha_{m},-\alpha_{m}\right], \alpha_{m}$ is a given maximum angle; 4-Fracture numbers in a unit area is noted as fracture density. Figure 5a-d show the connective status when the average fracture length changes under different parameters.

Model scale varies widely, with a large range of fracture density. Besides, fracture length is also a key factor affecting penetration. Thus a parameter is required to incorporate the effects of these two factors. Robinson [5] once presented such a parameter $N_{\mathrm{c}}=$ $($ density $) \times(\text { length scale })^{2}$. But its value is unstable. In 
(a)

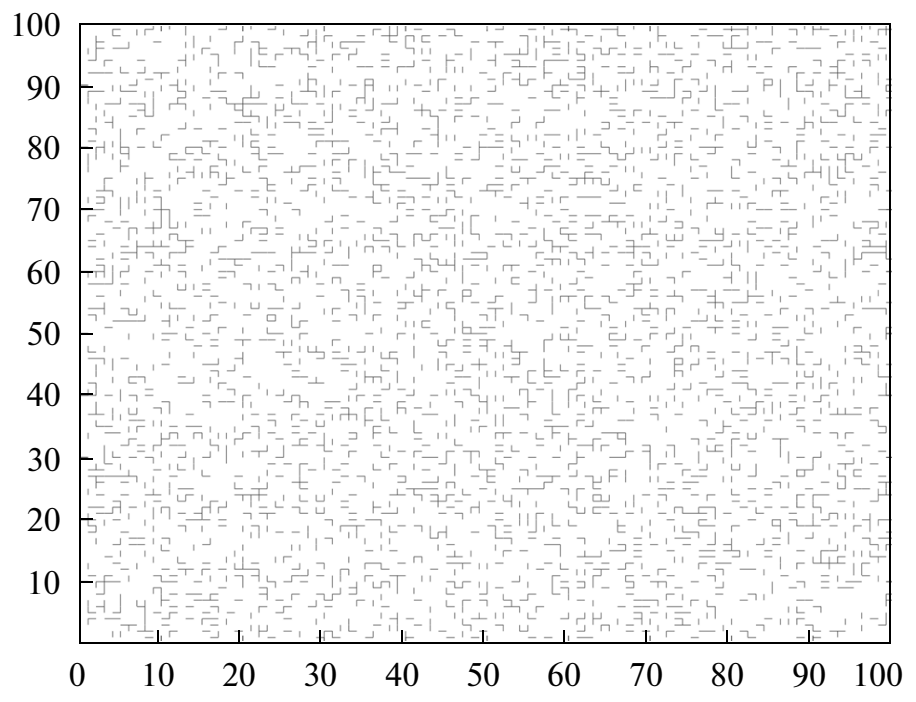

(b)

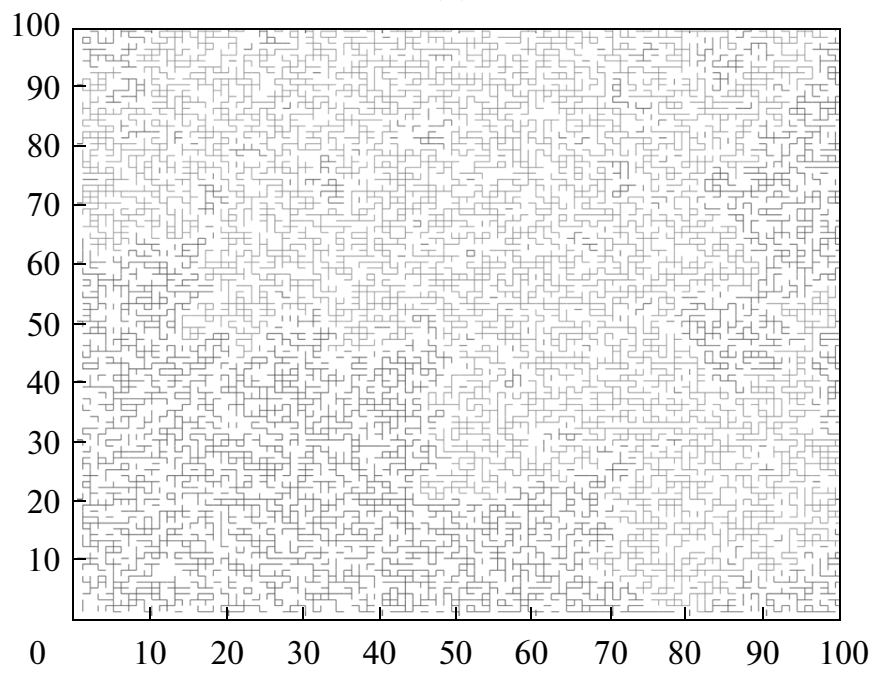

(c)

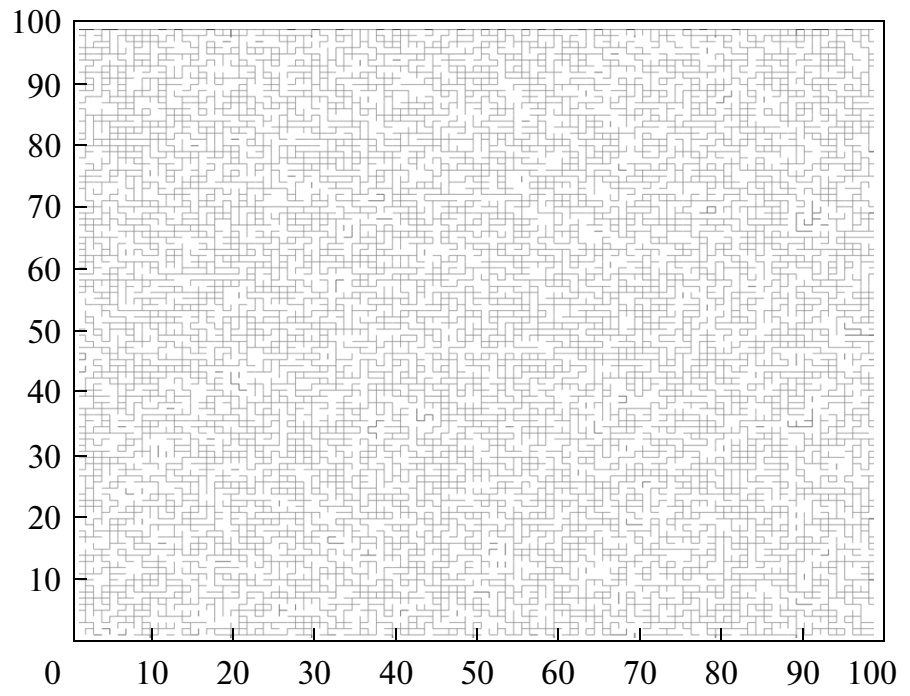

Fig. 2. The large percolation cluster expands with $p$. There is no large percolation cluster when (a) $p=0.2$, (b) the large percolation cluster initially appear at $p=0.5$ (light gray color), (c) the large percolation cluster expands at $p=0.6$. 


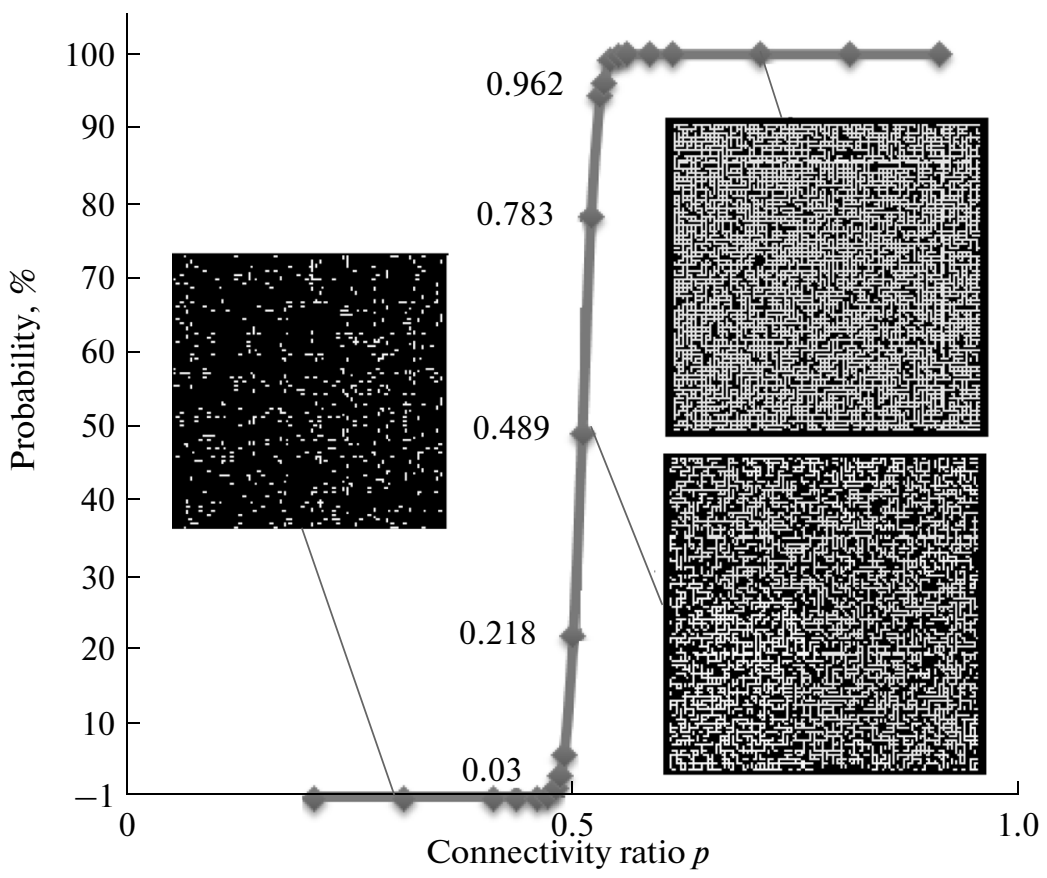

Fig. 3. Connectivity versus probability: Transverse coordinate is the connectivity ratio $p$, vertical coordinate is the probability when the large percolation cluster occurs at the scale of model $100 \times 100$ and repeated times of 100 (it is shown the percolation shreshold is 0.5 ).

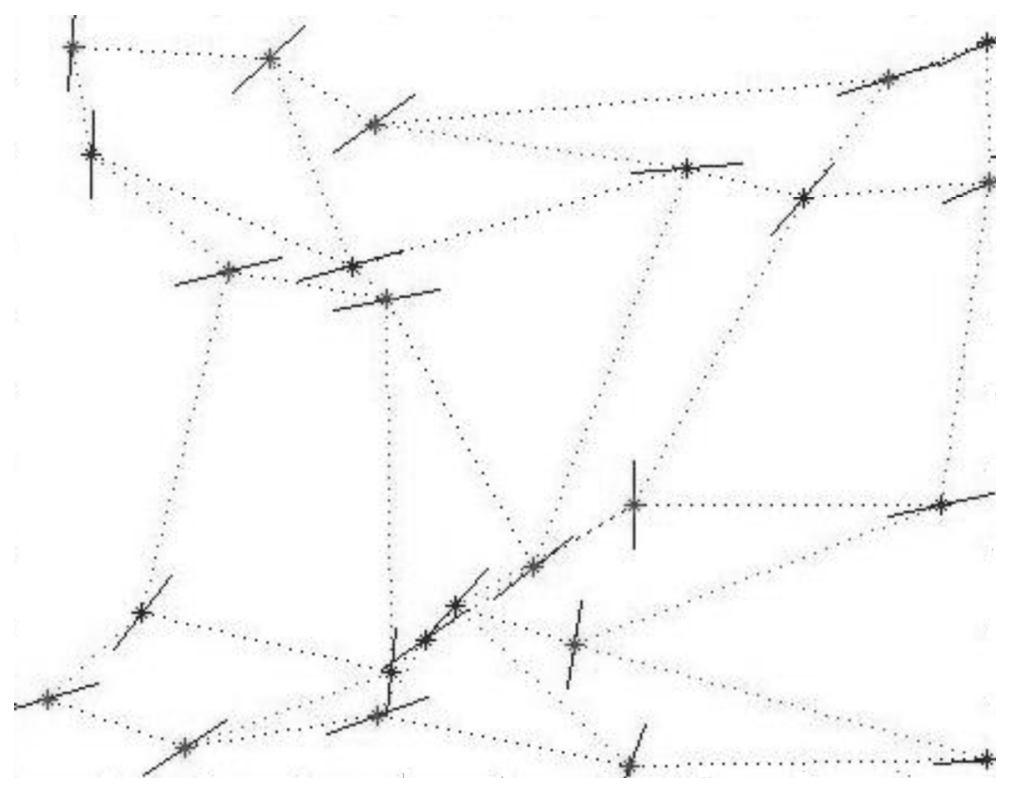

Fig. 4. Sketch of the network of percolation in porous and fractured dual-medium. 
(a)

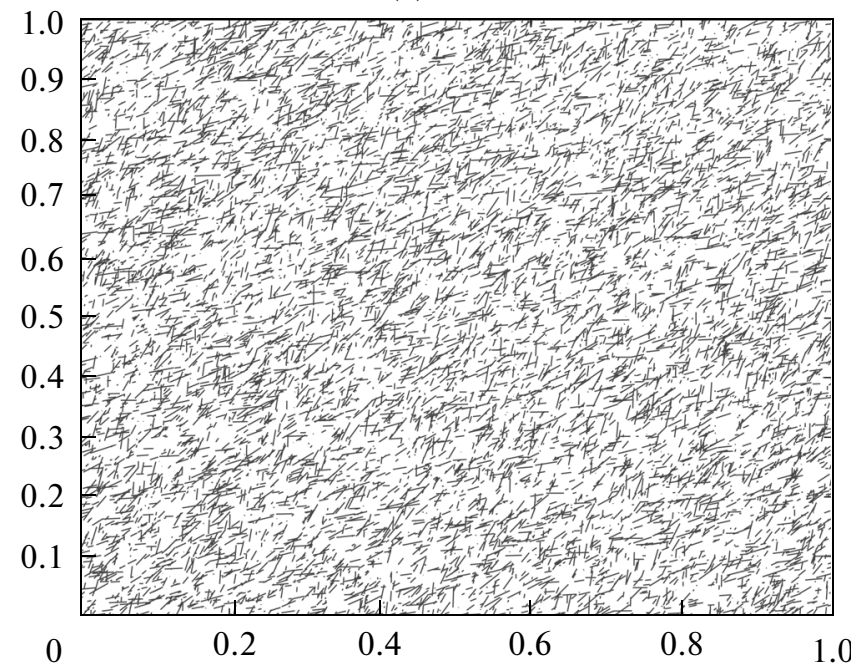

(b)

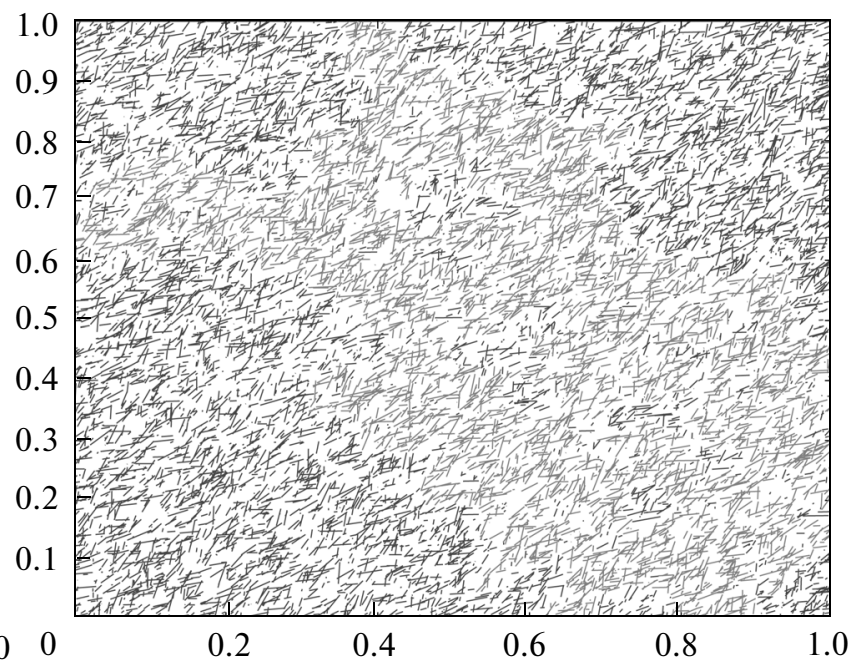

(d)

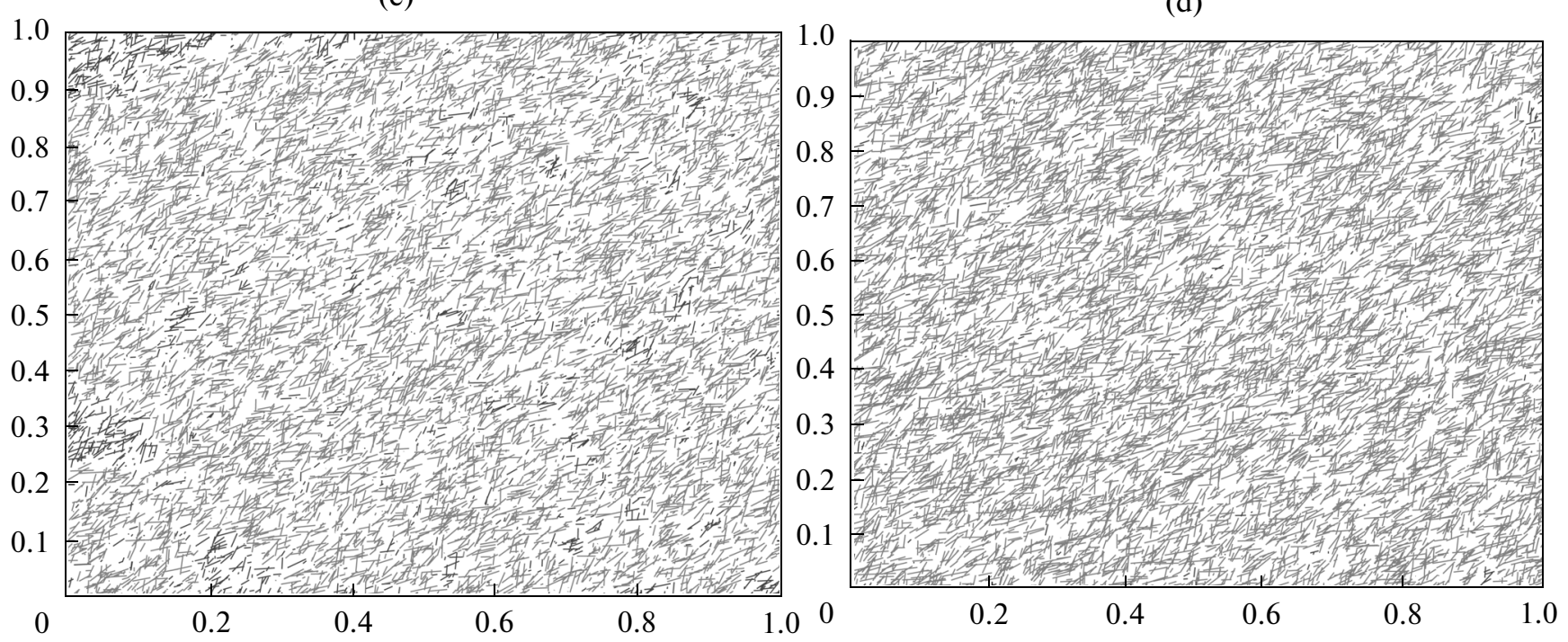

Fig. 5. Penetrated fractured cluster expands with $\mu$. (a) Average value $\mu=0.01$, mean square error $\sigma=0.01$, there is no penetrated fracture cluster; (b) penetrated fractured cluster occurs $(\mu=0.013, \sigma=0.01)$; (c) penetrated fractured cluster increase $(\mu=0.015$, $\sigma=0.01)$; (d) penetrated fractured cluster covers the whole zone $(\mu=0.02, \sigma=0.01)$.

Average CPU time in each computation under different fracture density

\begin{tabular}{l|c|c|c|c|c|c|c|c}
\hline Fracture Density & 100 & 400 & 900 & 1600 & 2500 & 4900 & 10000 & 12100 \\
\hline CPU Time, s & 0.016 & 0.078 & 0.31 & 0.83 & 2.05 & 7.31 & 28.5 & 40.7 \\
\hline
\end{tabular}

* Computation is processed in a personal computer with a CPU of Intel having duo Cores (E8400) and a RAM with 3.0 GHz and a DDRII (800) with 3 GB 
(a)

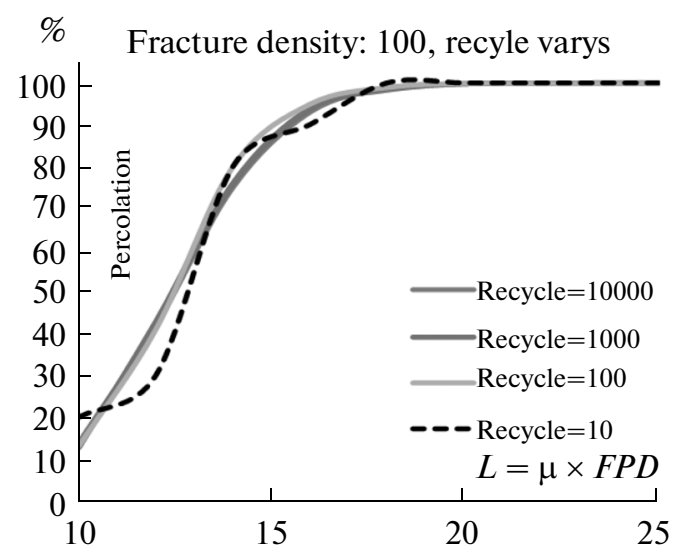

(c)

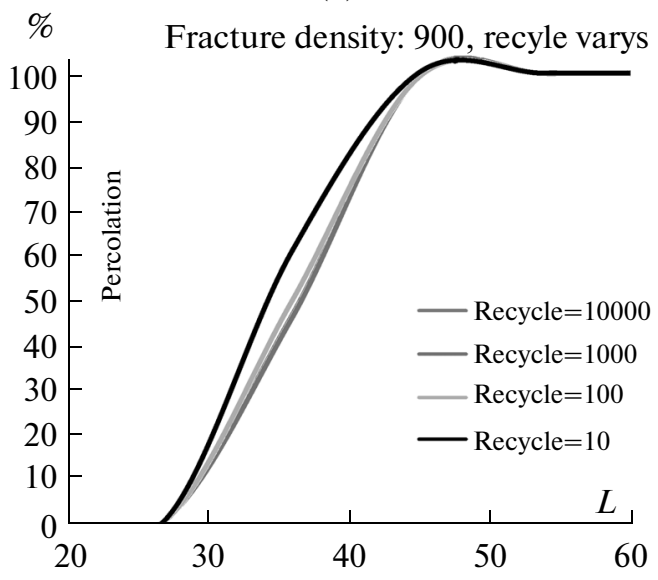

(b)

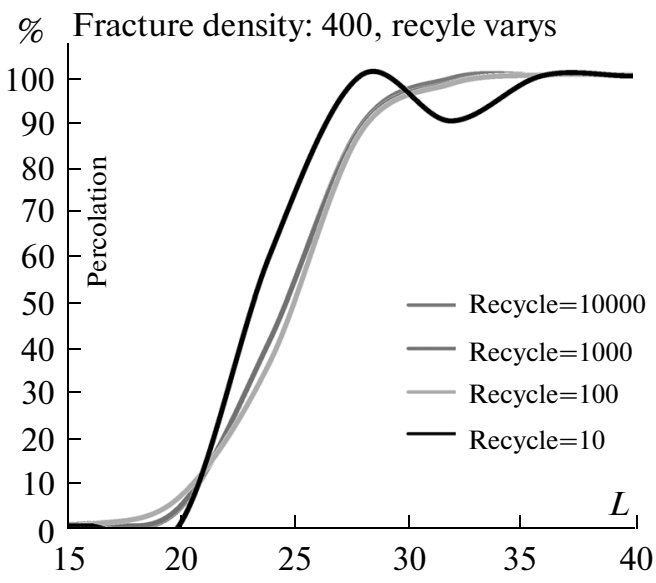

(d)

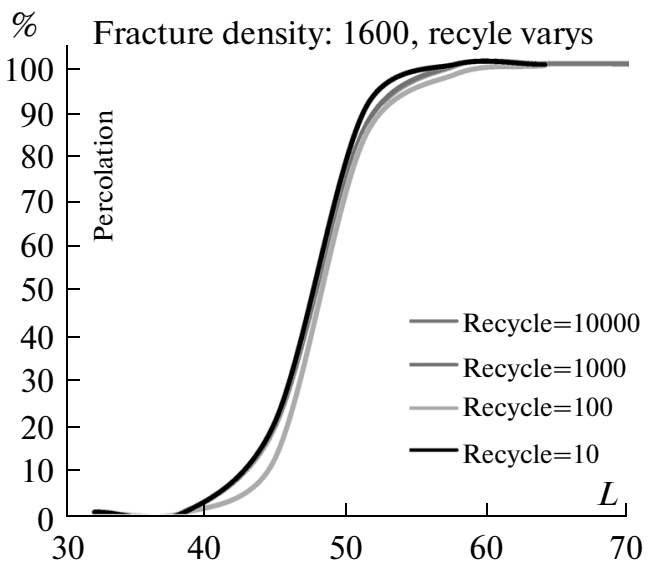

Fig. 6. Percolation varied with fracture density. Fracture number (a) 100, (b) 400, (c) 900, (d) 1600.

this paper, we modify the parameter as $N=$ density $\times$ $\mu^{x}$, here $x$ is a constant.

\section{NUMERICAL SIMULATIONS}

\section{Effects of Repeated Computation Times on the Random Error}

For a given fracture number, computation is often repeated to decrease the error. Generally, the more the repetition times and the fracture number, the smaller the random error. But this is time costing (table).

The effects of repetition on the random error are first analyzed at fracture number of 100, 400, 900, and 1600 , respectively. The $L$-axis indicates the total length of all fractures within the computational zone ( $L=\mu \times$ density) and the vertical axis (Penetration) indicates the ratio of times of penetrating fractures to the times of computation. The curves fluctuate at 10 repetitions and the fluctuation decreases as fracture number increases. But the differences are so small that the curves almost coincide when repetition varies from 100 to 10000 . Then the curves can be taken as reference. The fluctuation is found to decay with the rise of fracture number. For samples, the fluctuation falls into one wavelength when the fracture number is bigger than 900 and the curves coincide when the fracture number is bigger than 1600, as shown in Figs. 6a-d.

\section{Controlling Parameter $N$}

Besides the fracture number, the total fracture length $L=\mu \times$ density is also influential. However, its value is unstable when others change. Instead, we modify the Robinson parameter as $N=\mu^{\alpha} \times$ density, which incorporates the effects of the average fracture length and the fracture number (density) and thus more adoptable to describe the initial condition of penetrated fracture cluster.

In the Figs. $7 \mathrm{a}-\mathrm{f}, x$-coordinate $\alpha$ denotes the allowable maximum angle of fractures deviated from 
(a)

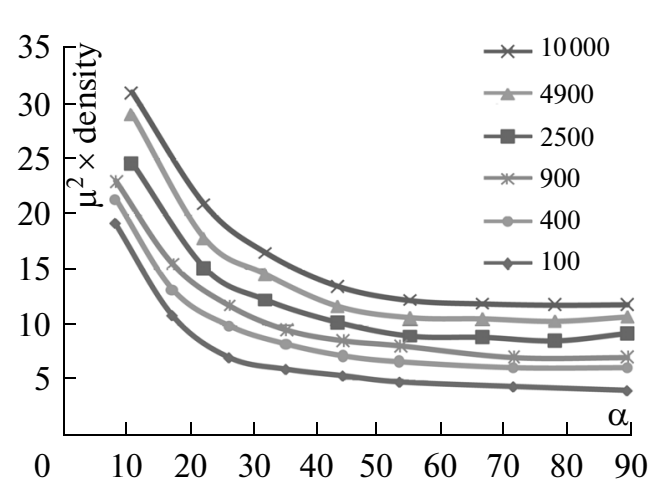

(c)

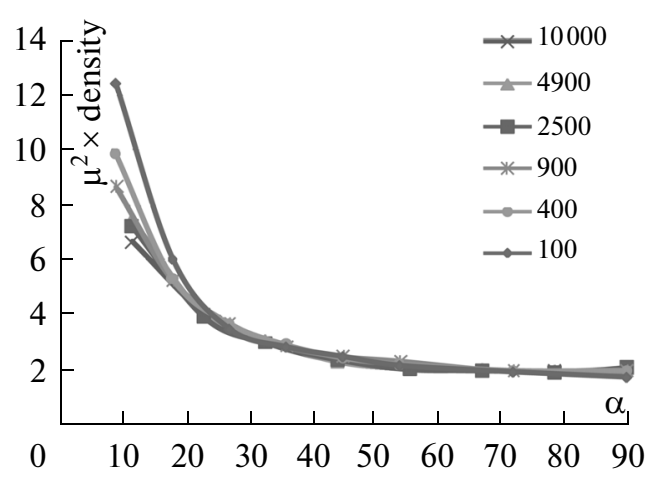

(e)

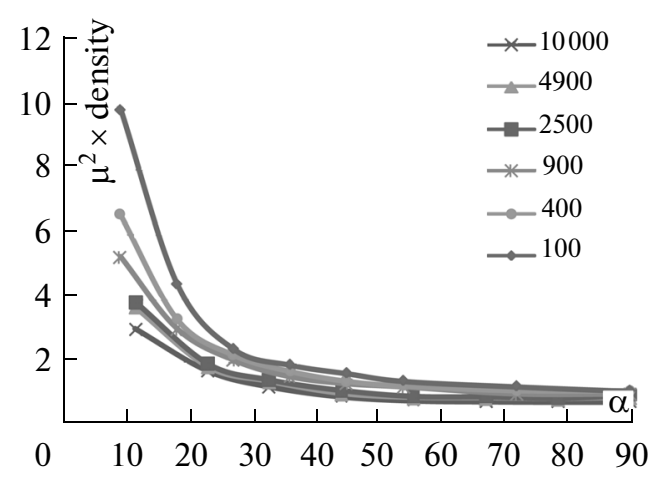

(b)

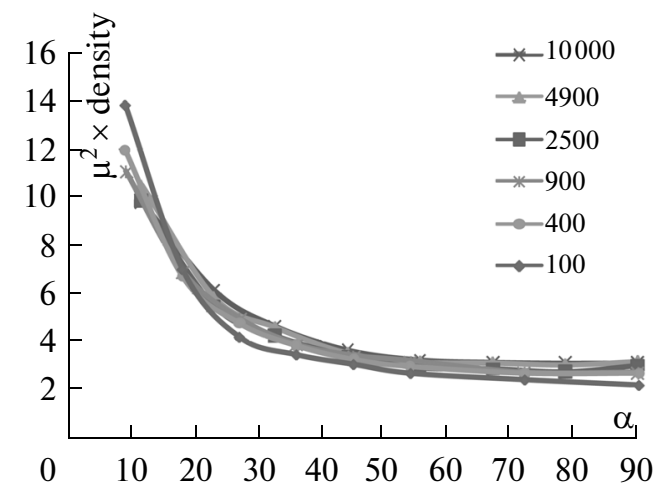

(d)

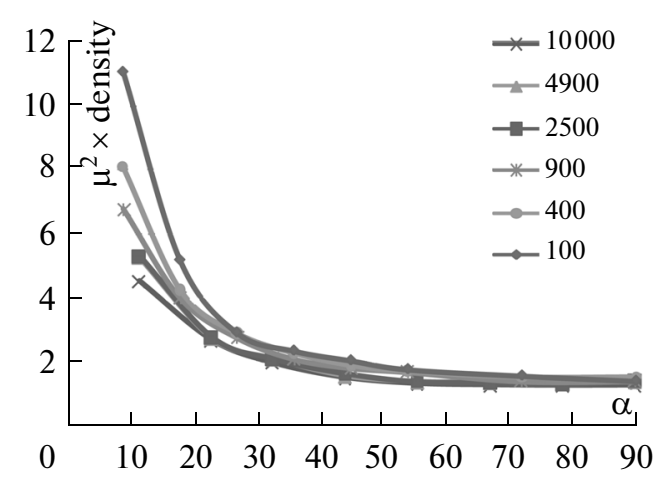

(f)

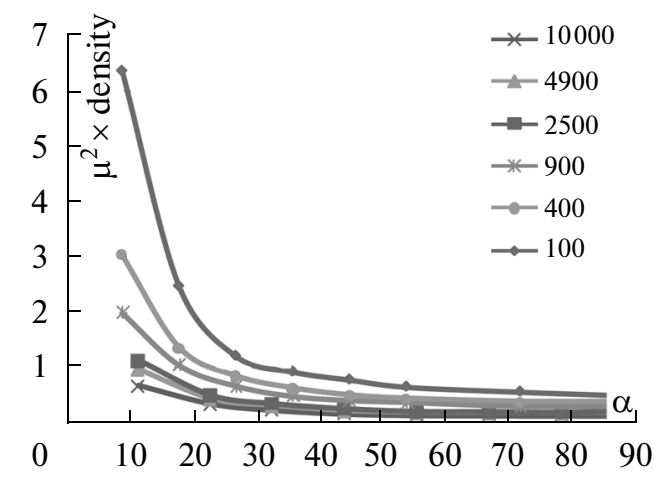

Fig. 7. The relationship of $N$ to the deviation angle of fracture $\alpha$ and a designed fracture density. (a) $N=\mu^{1.5} \times$ density, (b) $N=$ $\mu^{1.8} \times$ density, (c) $N=\mu^{1.9} \times$ density, (d) $N=\mu^{2.0} \times$ density, (e) $N=\mu^{2.1} \times$ density, (f) $N=\mu^{2.5} \times$ density.

horizontal direction. $Y$-coordinate denotes the parameter $N$ corresponding to the maximum deviation angle of fracture $\alpha$ and a designed fracture density. Each point in the figures is corresponding to the "final results" obtained by repeated computation under different fracture lengthes at given fracture density and maximum deviation angle. The "final results" are the value of $N$ corresponding to the threshold.
When parameter $N=\mu^{1.9} \times$ density is adopted, the curves tend to coincide. So $N$ can be taken as the control parameter, as shown in Figs. 7a-f. The curves with different fracture densities are close with each other (Fig. 7c); and they are almost coincident when $\alpha>$ $20^{\circ}$. When $\alpha<55^{\circ}$, the required $N$ increases fast corresponding to the occurrence of penetrated cluster. That means the required average fracture length 


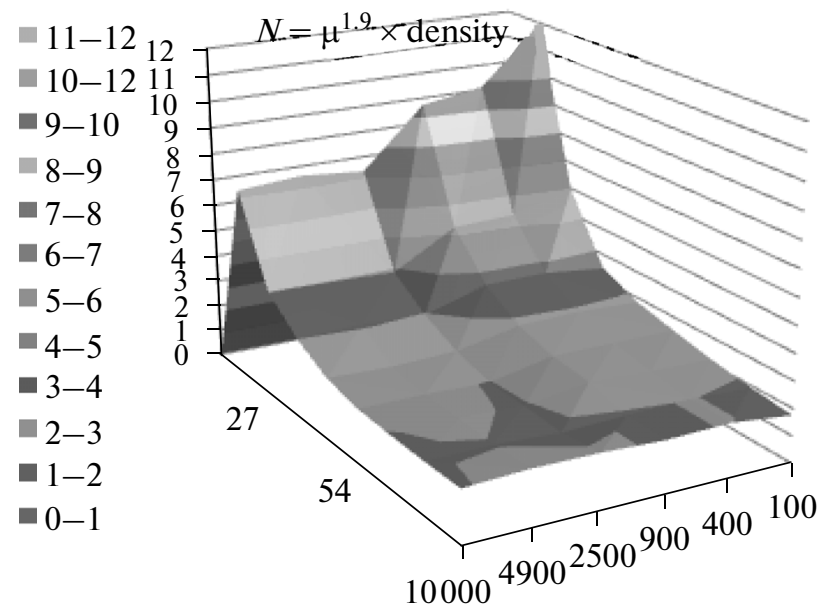

Fig. 8. Three dimensional $N \sim \alpha$ fracture density.

increases fast. However, when $\alpha>55^{\circ}$, the required $N$ decreases. If the data in Fig. 7c are shown in threedimensional surface, we can find that $N$ decreases with the increase of fracture density (Fig. 8). When $\alpha$ is about $10^{\circ}, N$ varies much at different fracture densities; and it varies little when $\alpha$ is about $80^{\circ}$. When $\alpha>$ $55^{\circ}, N$ is very close for different $\alpha$. The results with large fracture density can be obtained from those with small density. To investigate the characteristics of $N$ further, $\alpha$ is adopted as $67^{\circ}, 78.5^{\circ}$ and $90^{\circ}$ in computation, respectively. We can find that $N$ is close to 2.0 with the changes of fracture density and $\alpha$ (Fig. 9). Therefore, the value 2.0 can be taken as the threshold that penetrating fracture cluster occurs in fractured porous dual media system.

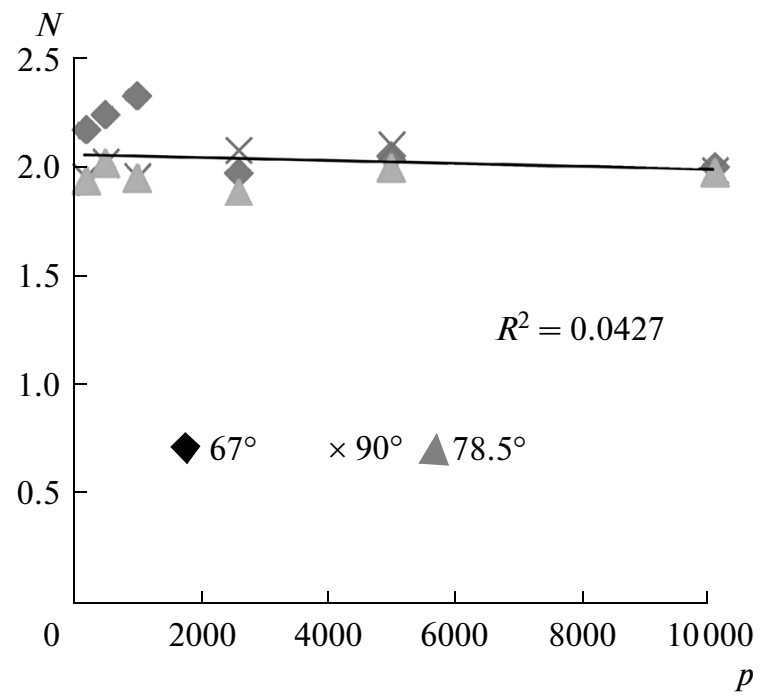

Fig. 9. $N \sim \alpha$ corresponding to different fracrture densities.

\section{Fractal Characteristics of Dual Percolation Model}

Fractured porous media has obvious fractal characteristics. Kang et al. [23] found that natural fractured media obeyed power law: $N_{\mathrm{p}}(\lambda)=A_{0} \lambda^{-D}$, in which $N_{\mathrm{p}}(\lambda)$ is the number of the fractures with a length of $\lambda, A_{0}$ is a coefficient, $D$ is the fractal dimension. For fractured network model, Robinson [5] introduced the parameter $N_{\mathrm{c}}=F P D \times L_{\mathrm{c}}^{2}$, with $F P D$ the fracture density and $L_{\mathrm{c}}$ the character length, to estimate the connectivity. The exponent 2 can be taken as the character fractal dimension corresponding to the fracture network. We find that the fractal dimension is 1.9 when the connectivity is 0.2 and the direction of fractures are highly disordered $\left(\alpha=90^{\circ}\right)$ Dealing with the numerical results according to the equation $A_{0}=F P D \times \mu^{D}$ (Fig. 10).

\section{Physical Meaning of Parameters $A_{0}$ and $D$}

Previous studies on connectivity focus on variation of parameter $A_{0}$ at $D=2 . A_{0}$ denotes either the average fracture length of the fracture network [5] or the impact range of the connected bonds in the porous percolation network [24-26]. There are no discussions on why $D$ is 2 and its meaning. This paper discusses the physical meaning of the fractal dimension $D$ in the formula $A_{0}=F P D \times \mu^{D}$ describing the dual percolation model $(F P D$ is the fracture density in unit area).

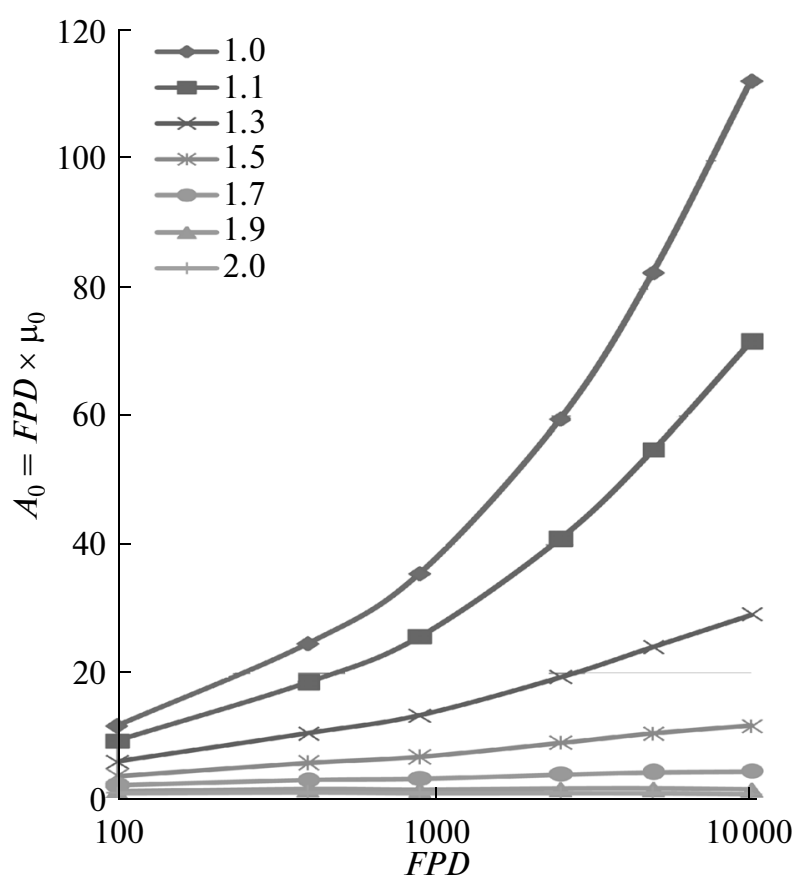

Fig. 10. Relationship between the coefficient and FPD under different fractal dimension. 


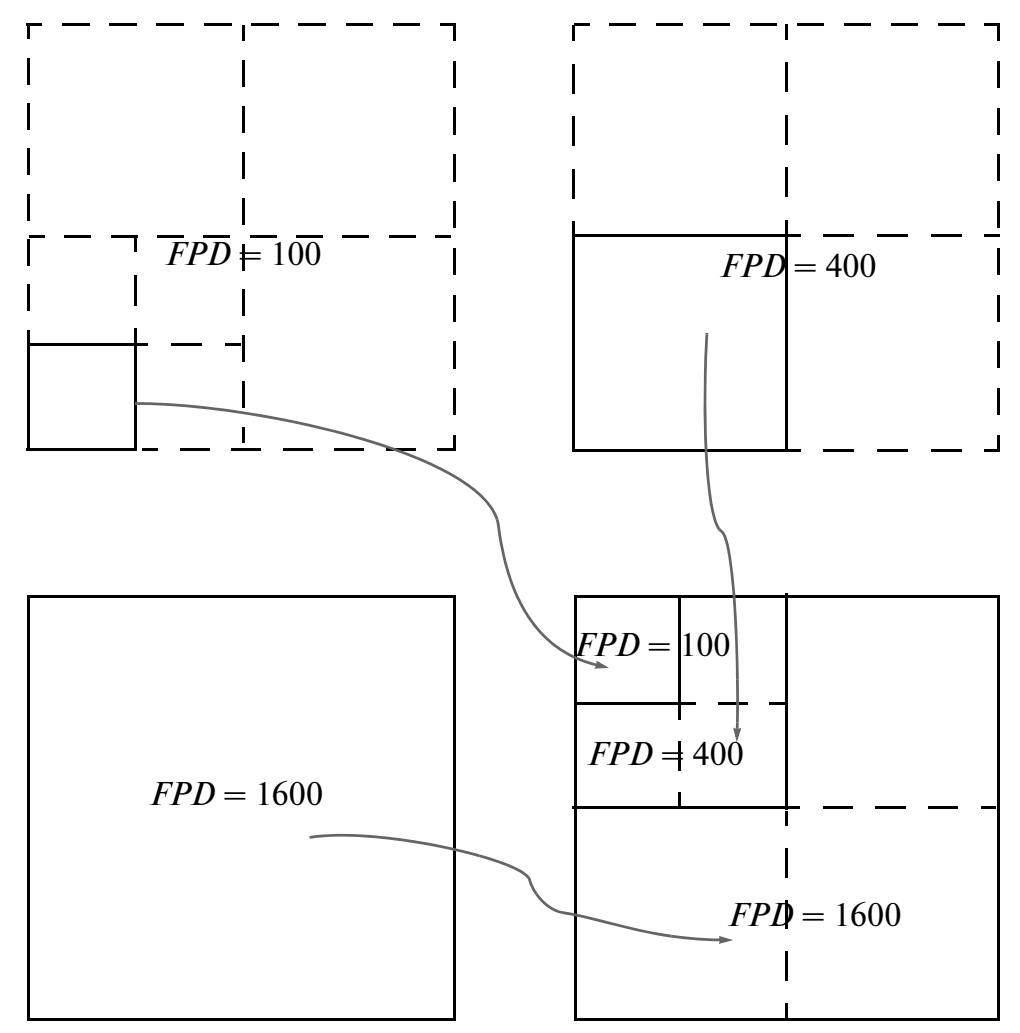

Fig. 11. Sketch of computation zone considering the corresponding scales.

In the dual percolation model, the real size of the concerned zone corresponds to the character length $\lambda$. The average length of the fractures is $\mu=\delta / \lambda$. Therefore, the computational zone is abstracted as $1 \times 1$ while the average length of the fractures is in the range of $0<\mu \leq 1$. The fractal dimension $D$ has only statistical meaning. Given a fractured porous media, the distribution and parameters such as $\mu$ and $\sigma$ of fracture and pore are determinate. The fracture density doesn't change obviously at the same character scale; the change of fracture density in a zone $(1 \times 1)$ reflects only the change of character length $\lambda$. The following relation holds for $F P D$ and $\lambda_{i}$ :

$$
F P D=C_{1}^{2} \lambda_{i}^{2},
$$

where the coefficient $C_{1}^{2}$ is a constant, $\lambda_{i}^{2}$ is the character length of the computational zone when FPD equals $i$ (Fig. 11). When the whole fracture network is fixed, the following relations are satisfied:

$$
4 \lambda_{100}=2 \lambda_{400}=\lambda_{1600}, \quad \mu_{i}=\delta_{i} / \lambda_{i} .
$$

1 -If $D=2$, then $A_{0}=F P D \times \mu^{D}=\left(C_{1} \lambda_{i}\right)^{2}$. Since $A_{0}$ is a constant, $\delta_{i}$ is also a constant. Thus this equation is always correct if only it is correct for any FPD. For an example, the connectivity at $F P D=100$ is the same with that at $F P D=1600$. In other words, the connectivity in a zone with area $(10 \mathrm{~m})^{2}$ is the same as that with area $(40 \mathrm{~m})^{2}$ if the real sizes of the computing zone are $10 \times 10$ and $40 \times 40 \mathrm{~m}$, respectively Thus the connectivity of small zone can reflect that of large zone. The fractured porous media satisfied this type of characteristics is called "critical" fractured porous media.

2-If $D<2$, then $A_{0}=F P D \mu^{D}=\left(M_{1} \lambda_{i}\right)^{2-D} \times$ $\left(M_{1} \delta_{i}\right)^{D}$. Since $A_{0}$ is a constant, and $2-D>0, \delta_{i}$ is inversely proportional to $\lambda_{i}$. For the sample in (1), because of $\lambda_{1600}>\lambda_{400}>\lambda_{100}$, the percolation threshold value $\delta_{1600}>\delta_{400}>\delta_{100}$, meaning that the connectivity in the zones with areas $(40 \mathrm{~m})^{2},(20 \mathrm{~m})^{2}$ and $(10 \mathrm{~m})^{2}$ decreases in order. For such a case, the connectivity is more likely to occur when more fractures are involved in the concerned zone. And the computing connectivity for small zone does not reflect that in large zone. Such a rock is unfavorable for the closure of strata; and the media with fractures in such a distribution may be called the "dispersion" fractured porous media.

3-If $D>2$, then $A_{0}=F P D \mu^{D}=\left(M_{1} \delta_{i}\right)^{D} /\left(M_{1} \lambda_{i}\right)^{D-2}$. Since $A_{0}$ is a constant and $D-2>0, \delta_{i}$ is inversely proportional to $\lambda_{i}$. Adopting the example in (1), because of $\lambda_{1600}>\lambda_{400}>\lambda_{100}$, the corresponding percolation threshold values satisfy $\delta_{1600}>\delta_{400}>\delta_{100}$. That means, the connectivity satisfy in zone $(40 \mathrm{~m})^{2}<$ in zone $(20 \mathrm{~m})^{2}<$ in zone $(10 \mathrm{~m})^{2}$. For such a case, the 

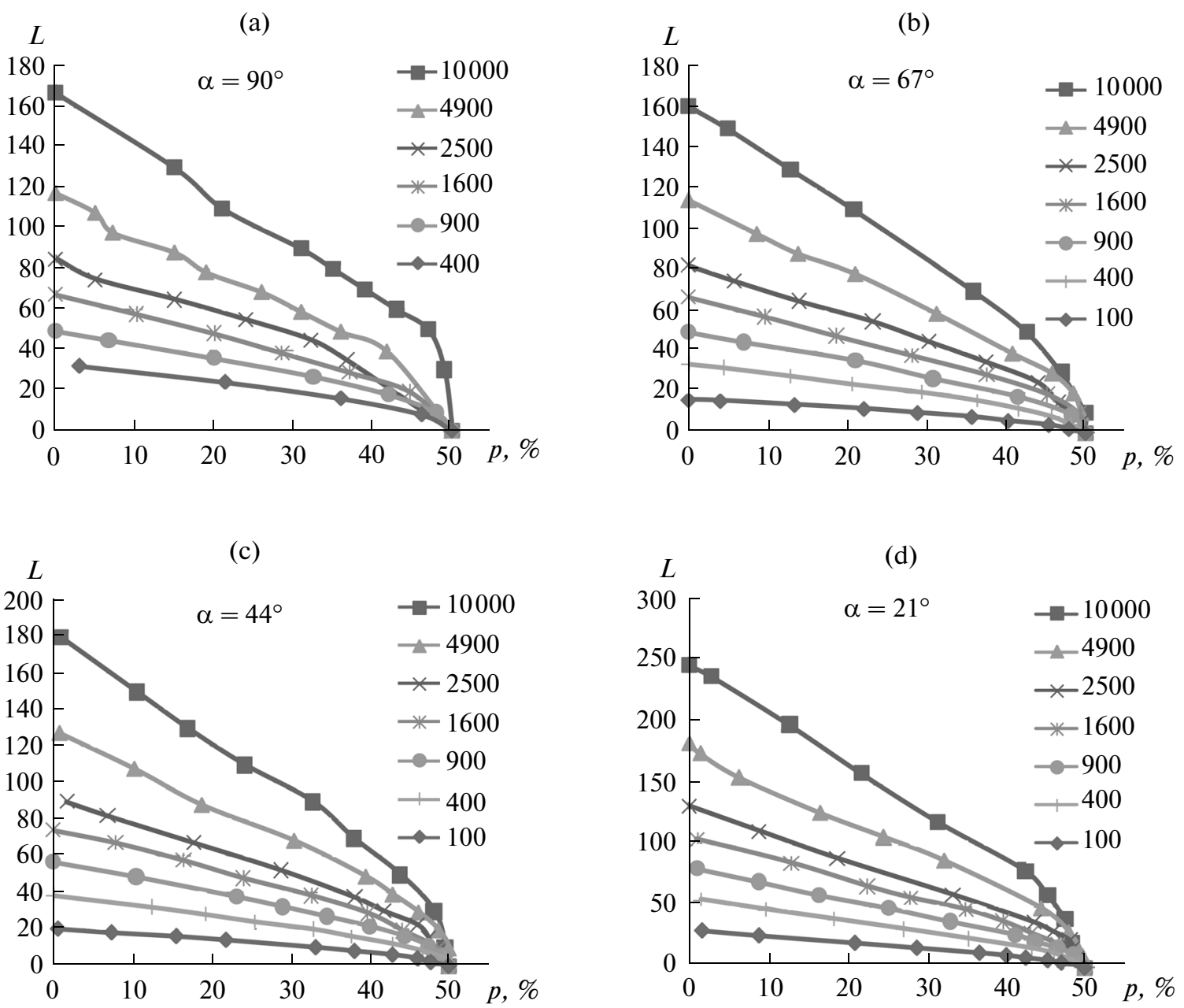

Fig. 12. The relationship between $L$ and $p$ under different $\alpha$ : $90^{\circ}$ (a), $67^{\circ}$ (b), $44^{\circ}(\mathrm{c}), 21^{\circ}(\mathrm{d})$.
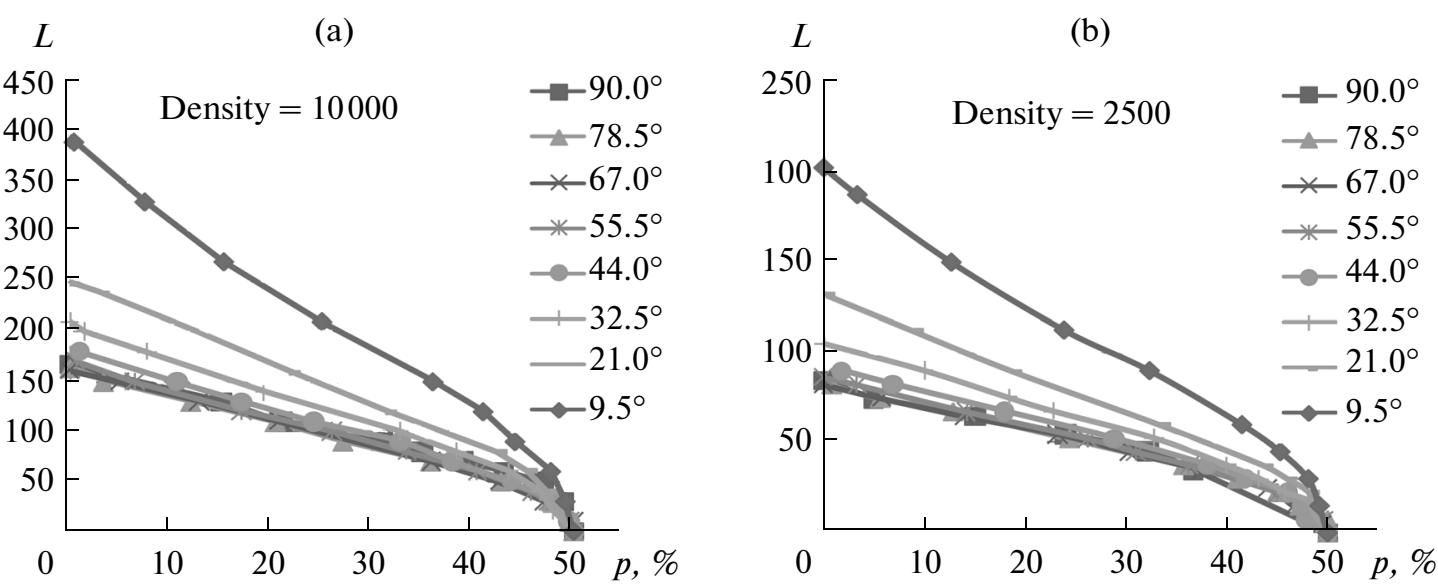

Fig. 13. Curves of $L \sim p$ under different fracture angles when fracture denstity $=10000$ and 2500. Curves of $L \sim p$ when fracture density $=10000(a), 2500(b)$. 

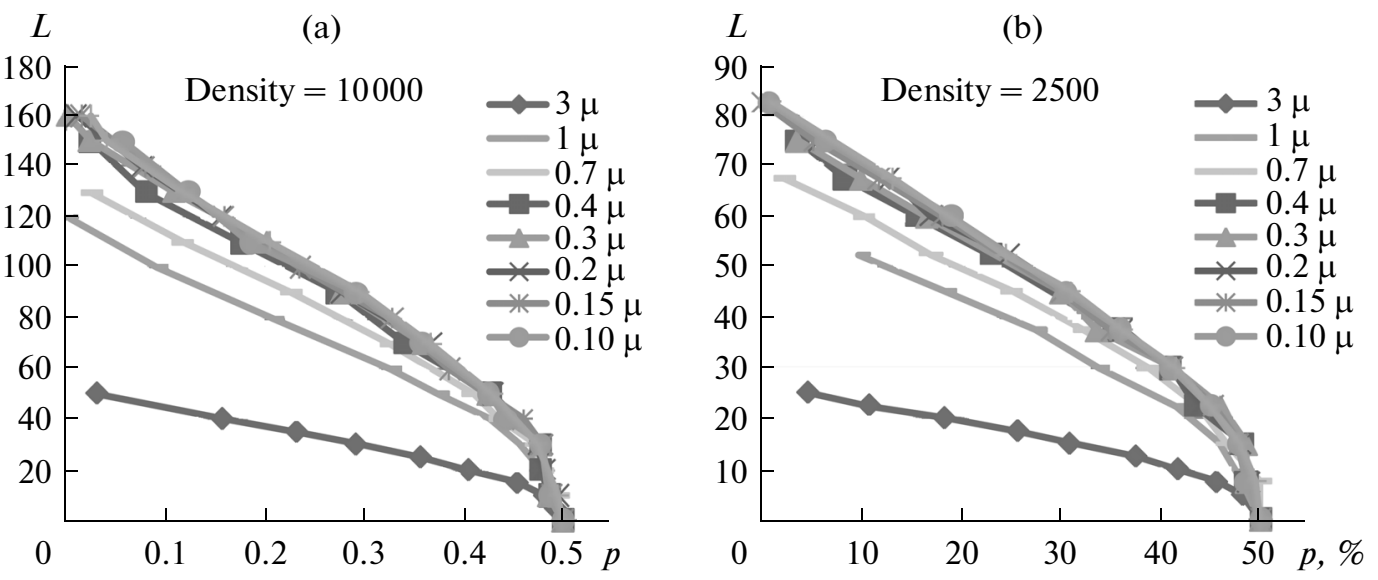

Fig. 14. Curves of $L \sim p$ under different fracture $\mu$ when fracture denstity $=10000$ and 2500 . When fracture density $=10000$, variance of fractures and probability $L \sim p$ (a), when fracture density $=2500$, variance of fractures and probability $L \sim p$ (b).

connectivity is more unlikely to occur when more fractures are involved in the concerned zone. On the contrary, for a small zone with fewer fractures, it tends to connectivity. For this kind of rock, connectivity calculation in small zone, if satisfying the condition, can ensure the connectivity in large scale. Such a rock is favorable for the closure of strata; and the media with fractures in such a distribution may be called the "directional type" fractured porous media.

\section{RESULTS AND DISCUSSION}

During computing, the dual percolation network is considered as connected (The corresponding parameters in this case is called the combinational percolation ones) if there exists any fracture satisfying that it forms a path to and joins the other end.

The Monte-Carlo method is used in computation. In each computation, 50 100 samples are adopted to compute the probability of connectivity under different combination of parameters (FPD, $p, \alpha, \mu, \sigma)$. The combination of parameters are taken as the threshold when the probability of connectivity arrives at $50 \%$, then $f_{\mathrm{c}}(F P D, p, \alpha, \mu, \sigma)=0$.

The percolation threshold $f_{\mathrm{c}}(F P D, p, \alpha, \mu, \sigma)$ can be written in the form of $f_{\mathrm{c}}\left(\left(\left[A_{0}, D\right], p, \alpha, \mu, \sigma\right)_{\mathrm{c}}\right)$ by using the expression $A_{0}=F P D \times \mu^{D}$. The results in the next sections are obtained according to different combination of $\left(\left[A_{0}, D\right], p, \alpha, \mu, \sigma\right)_{\mathrm{c}}$.

\section{Effects of Connective Probability $p$ of the Network on the Percolation}

The $L-p$ relationship is considered for different fracture densities under four cases of $\alpha$, as shown in Figs. $12 \mathrm{a}-\mathrm{d}$, where $p$ indicates the connectivity probability of "bond" in dual percolation model, which reflects the developing degree of macro pores and defects with strong connection potential surrounding small fractures; and $L(L=\mu \times$ density $)$ is the total length of fractures in the computation zone when connective cluster reaches $50 \%$ of the total computation times at given $p, \alpha$, and fracture density.

With the increase of connectivity of "bond" in the percolation network, the total length $L$ for the requirement of penetrating cluster's formation decreases linearly till the probability is close to $45 \%$, afterwards, the decrease of $L$ becomes fast. The required total fracture length increases with the increase of fracture density for all possible $p, \alpha$.

When $\alpha$ changes, $L \sim p$ can be divided into linear and nonlinear parts. When $\alpha>55^{\circ}$, the curves coincide, corresponding to the relation $N \sim \alpha$. It indicates that the characteristics of the sample with 10000 fractures can be simulated by a sample with 2500 fractures, as shown in Fig. 13a, b.

Figures $14 \mathrm{a}, \mathrm{b}$ show the relation of $L \sim p$ under different variance of fracture length when $\alpha=90^{\circ}$ and the fracture density is fixed. The data adopted in computation are as follows: the fracture densities are 10000 and 2500, respectively, the variance of fracture length changes from 0.1 to $3.0 \mu$ ( $\mu$ is the average fracture length). The linear and nonlinear parts of the curve $L \sim p$ are obvious. The inflexion is at $45 \%$. When the variance of fracture is below $0.4 \mu$, the curves are almost coincided. However, when the variance of fracture is larger than $0.7 \mu$, the curves are deviated obviously.

\section{Results under Equal-Length Fractures}

To investigate the effects of $\alpha$ and $p$ on $D$ and $A_{0}$, numerical simulations are carried out under different 
(a)

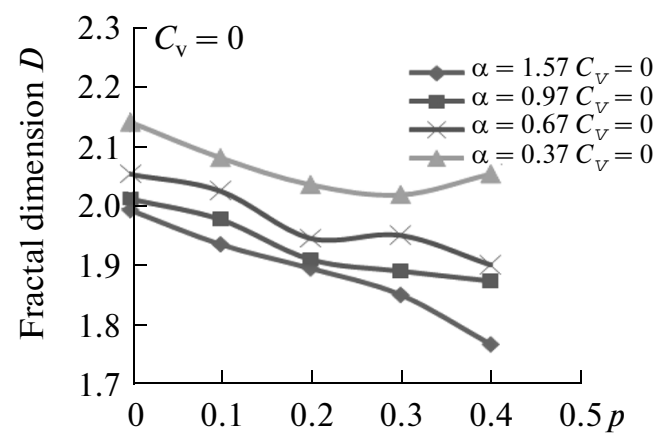

(c)

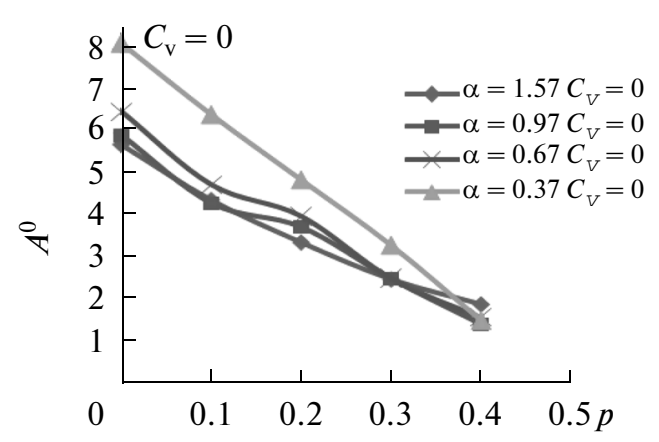

(b)

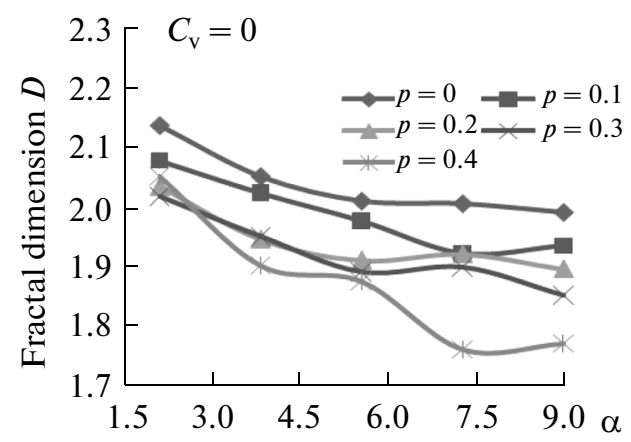

(d)

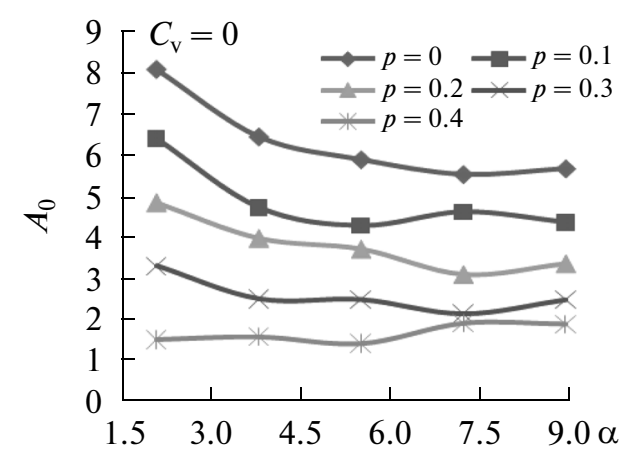

Fig. 15. Development of $D$ and $A_{0}$ with $\alpha$ and $p$ at $C_{V}=0$ : development of $D$ with connective probability $p$ under different maximum angle of fracture $\alpha$ (a), with maximum angle of fracture $\alpha$ under different connective probability $p$ (b); development of $A_{0}$ with connective probability $p$ under different maximum angle of fracture $\alpha(\mathrm{c})$, with maximum angle of fracture $\alpha$ under different connective probability $p(\mathrm{~d})$.

values of $\alpha, p$ with fractures of equal-length (i.e., $C_{V}=$ $\sigma / \mu=0)$ and uniform distribution, and the angles between $[-\alpha, \alpha]$.

From the numerical results it follows that:

1-The fractal dimension $D$ decreases linearly with the increase of connective probability. That means the sediment tends to be "dispersion type" fractured porous media with the increase of $p$.

2-The fractal dimensions start to fluctuate obviously when the value is larger than 0.3 , which indicates that the porous percolation in the dual percolation network approaches the percolation threshold and so the threshold fluctuates by the effects of the equallength fractures when the porous connectivity is large.

3-The fractal dimension $D$ increases with the decrease of fracture angle $\alpha$. For example, the whole curve is above the line $D=2$ under $\alpha=0.37$, which shows that the dual percolation network has no characteristics of "dispersion type" porous fracture media and so this type of sediment is difficult to be connected.

4-The parameter $A_{0}$ decreases linearly with the increase of the connective probability $p$ under different fracture angle $\alpha$, which indicates that the required total length of fractures (i.e., the connectivity among fractures) to approach the threshold decreases with the increase of the porous connectivity.

5-The parameter $A_{0}$ decreases a little with the increase of the fracture angle $\alpha$ under the condition of $p<0.4$, which indicates that when the fractures' angles tends to be in the same direction, the corresponding sediment is difficult to be connected. The same values of $A_{0}$ under the condition of $p=0.4$ show that the porous connectivity controls the percolation of fractured porous media, as shown in Figs. 15a-d.

\section{Results under Non-Equal-Length Fractures}

In the computation of this section, the fractures satisfy the normal distribution $N_{\mathrm{n}}(\mu, \sigma)$. The fractures' angles range from $-90^{\circ}$ to $90^{\circ}$. The value of $C_{V}$ ranges from 0.0 to 3.0. The mean square error $\sigma$ is denoted by $C_{V}=\sigma / \mu$ considering that the value of $\mu$ changes obviously under different $F P D$.

The fractal dimension $D$ decreases linearly with increase of $p$. All values of $D$ fall between $D=-5.0 p+$ 2.01 and $D=-5.0 p+1.92$ with the fluctuation ampli- 
(a)

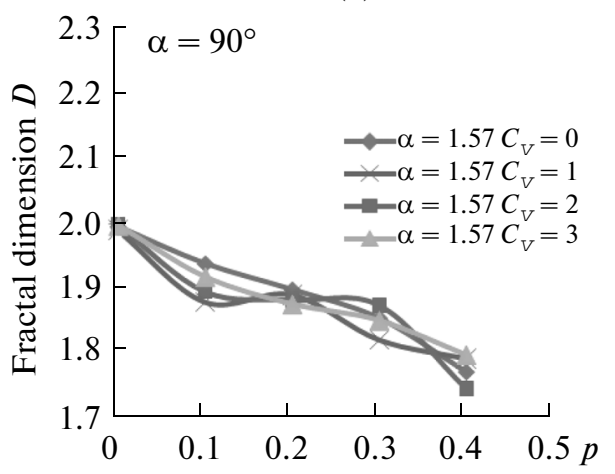

(c)

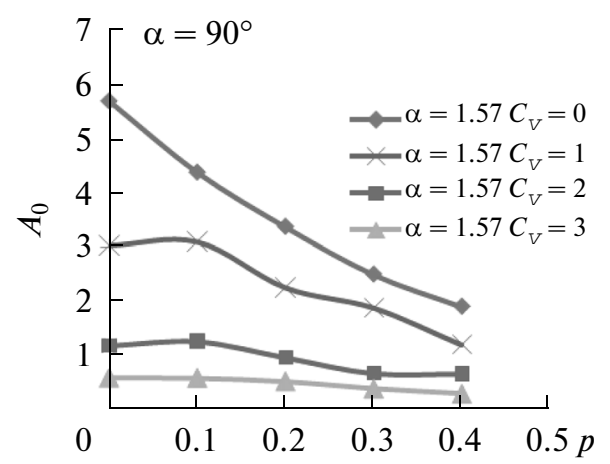

(b)

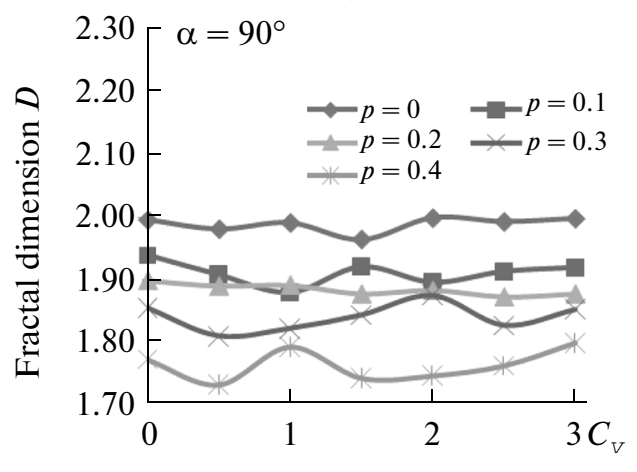

(d)

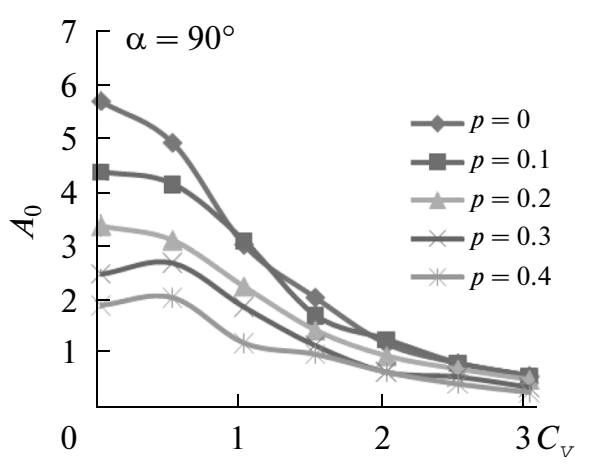

Fig. 16. Development of $D$ and $A_{0}$ with $C_{V}$ and $p$ at $\alpha=90^{\circ}$ : development of $D$ with connective probability $p$ under different maximum angle of fracture $C_{V}(\mathrm{a})$, with $C_{V}$ under different connective probability $p(\mathrm{~b})$; development of $A_{0}$ with connective probability $p$ under different $C_{V}(\mathrm{c})$, with $C_{V}$ under different connective probability $p(\mathrm{~d})$.

tudes between $\pm 2.5 \%$. And the fluctuation of $D$ increases with the increase of $C_{\mathrm{V}}$ under the condition of $\alpha=1.57\left( \pm 90^{\circ}\right) . D$ is always less than 2.0 for any $p$, indicating the "dispersion type" fractured porous media, as shown in Fig. 16a, b.

The position of $A_{0}$ lowers fast with the increase of $C_{V}$ (Fig. 16c). $A_{0}$ increases with the decrease of $p$ and this tendency is nearly linear at $C_{\mathrm{V}}=0$. At $C_{\mathrm{V}}>0$ and $p<0.1, A_{0}$ changes little, and at $C_{\mathrm{V}}>0$ and $p>0.1, A_{0}$ decreases linearly with the decrease of $p$.

$A_{0}$ decreases fast with the increase of $C_{\mathrm{V}}$ under the same $p$ (Fig. 16d). The reason is the occurrence of long fractures. The long fractures play an important role in percolation even if the sum of them is small. The larger the value of $C_{V}$, the more the long fractures, the stronger the controlling of long fractures to the connectivity. At this condition, even if only a few fractures are connected, the whole zone can be connected. That means the required average length may be decreased. On the other hand, the position of the curve $A_{0}$ becomes lower with the increase of $p$ because the increase of $p$ leads to the dominance of connectivity of pores.
The computing results considering the boundary effects when the fracture is distributed uniformly and the angles ranges randomly from $-73^{\circ}$ to $73^{\circ}$ and $C_{V}$ ranges from 0.0 to 3.0 show that the results are similar to that at $\alpha= \pm 90^{\circ}$, except that the curves of $A_{0}$ is a little higher. The values of $D$ range between the lines $D=$ $-5.0 p+2.12$ and $D=-5.0 p+2.12$. The differences of the average and the two lines are $\pm 3.0 \%$, as shown in Fig. $17 \mathrm{a}-$ d.

The characteristics of $D$ shown in Fig. $18 \mathrm{a}, \mathrm{b}$ are very similar to that in Fig. 15a, b. Although the media is "directional type" at $\alpha= \pm 21.1^{\circ}$, the distances between the $D \sim p$ curves are larger in Fig. 18a because of the uncertainty of fracture length. In Fig. 18c, the curves of $A_{0} \sim p$ tend to be coincided under different $\alpha$, which is different with that in Fig. 15c. Figure 18d shows that $A_{0} \sim \alpha$ curves keep stable under different connective probability $p$ while $A_{0}$ tends to decrease with $\alpha$ in Fig. 15d.

\section{CONCLUSIONS}

Fractured porous dual percolation is investigated in this paper. The threshold corresponding to initiation 
(a)

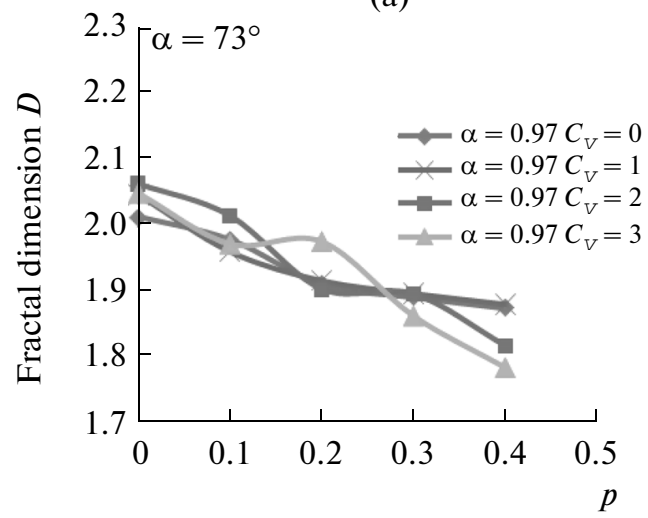

(c)

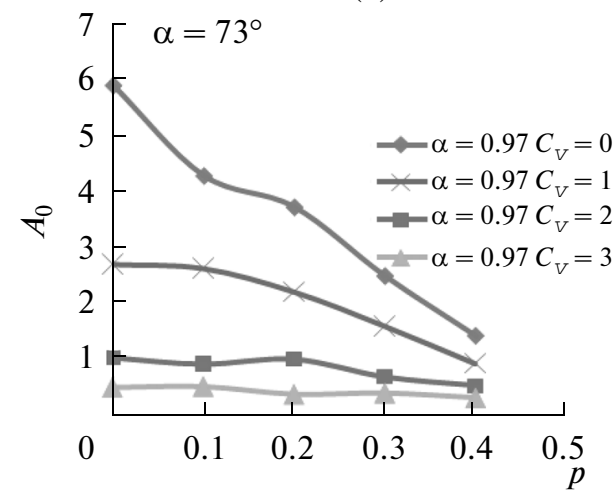

(b)

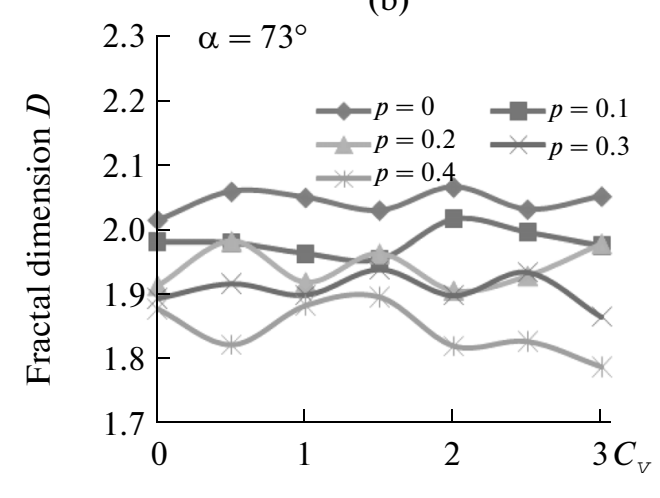

(d)

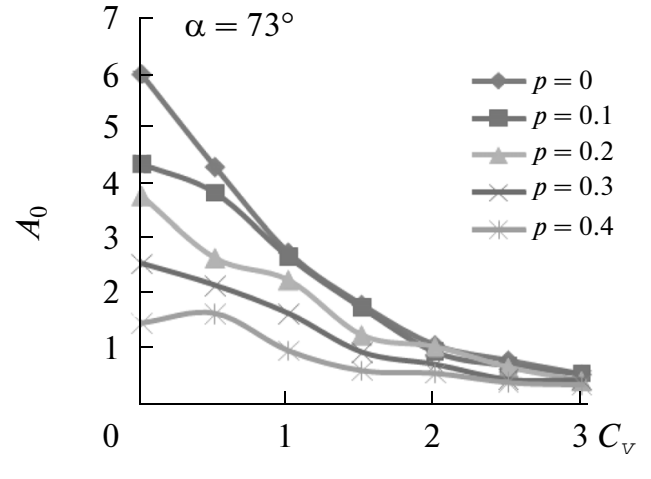

Fig. 17. Development of $D$ and $A_{0}$ with $C_{V}$ and $p$ at $\alpha=73^{\circ}$ : development of $D$ with connective probability $p$ under different $C_{V}$ (a), with $C_{V}$ under different connective probability $p$ (b); development of $A_{0}$ with connective probability $p$ under different $C_{V}$ (c), with $C_{V}$ under different connective probability $p(\mathrm{~d})$.

of penetrating fracture cluster is found. The main conclusions are as follows:

1 - The precise of results depend mainly on the scale of model and repetition times of calculation. The results are stable after 10 repetition of calculation when more than 1600 fractures are present. But this is limited by the cost of CPU, which increases exponentially with fracture density and linearly with repetitions. A more practical method is to increase the repetitions and decrease the fracture density.

2 - It is important to determine the parameter $N$ for the dual percolation analysis. Since $N$ is nearly constant at different fracture densities and the maximum deviation angle $\alpha$, the characteristics of model with big fracture density can be obtained from that with small fracture density (usually of 1600 2500). In this way, the cost of CPU time can be decrease considerably.

3-At the condition of $D=2$, the connectivity of small zone can reflect that of large zone. The fractured porous media satisfied this type of characteristics is called "critical" fractured porous media. At the condi- tion of $D<2$, the connectivity in a small zone doesn't reflect that in large zone. The rock with fractures in such a distribution may be called the "dispersion" fractured porous rock. At the condition of $D>2$, connectivity in a small zone can ensure the connectivity on large scale. The rock with fractures in such a distribution may be called the "directional type" fractured porous media.

4-When the connectivity of "bond" in the matrix percolation network arrives at $45 \%$, the percolation of the model becomes unstable and the threshold of the fracture for penetrating fracture cluster becomes small. That means, once the condition occurs, large channel of inter-porosity flow emerges.

5-Relationship of $L \sim p$ under different variances of fracture length shows that when the variance $\sigma$ is bigger than $0.5 \mu$, the $L \sim p$ curve decreases obviously after inflection point. That means, the connective fracture system will occur under small fracture parameters at this condition. 
(a)

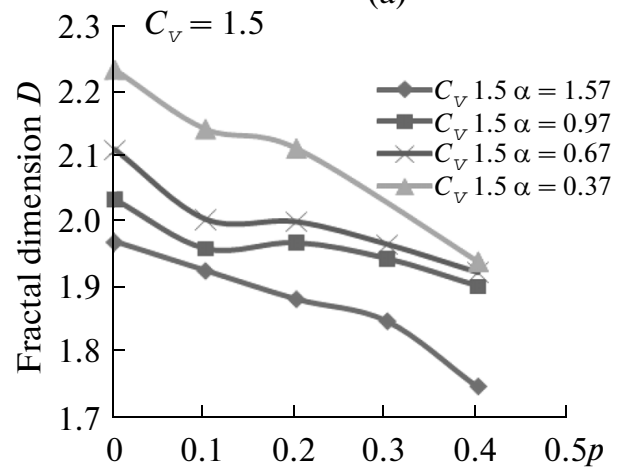

(c)

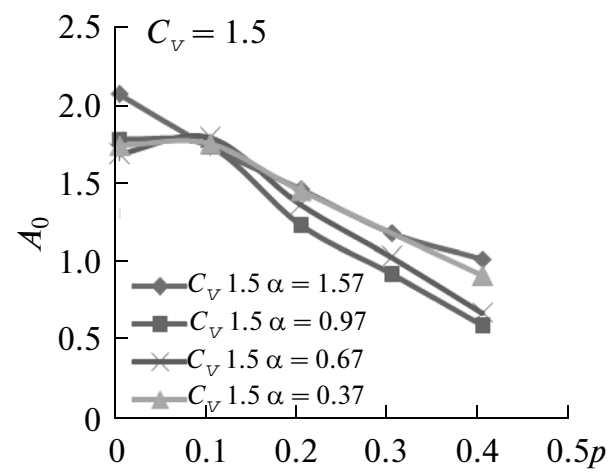

(b)

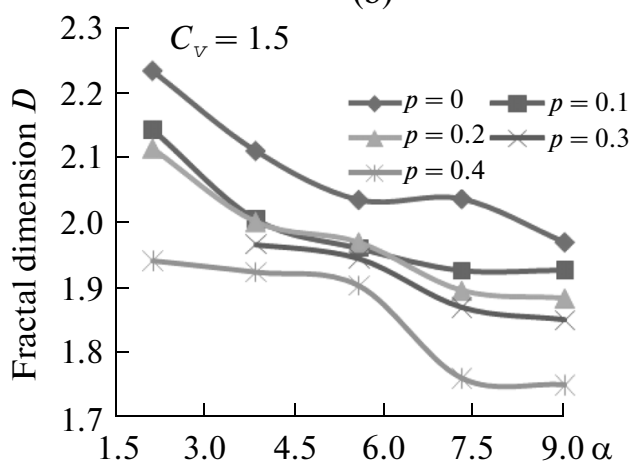

(d)

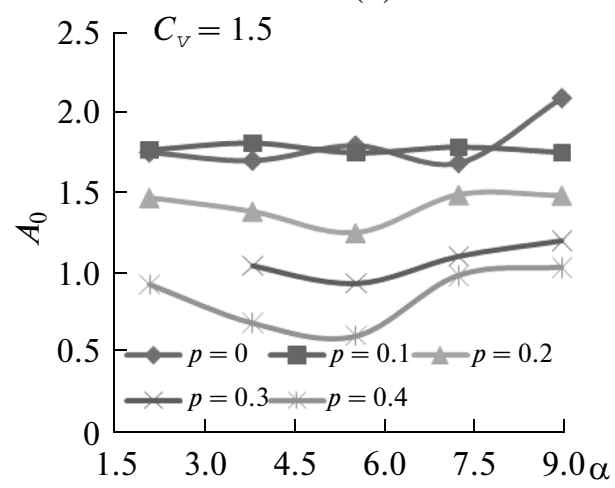

Fig. 18. Development of $D$ and $A_{0}$ with $\alpha$ and $p$ at $C_{V}=1.5$ : development of $D$ with connective probability $p$ under different maximum angle of fracture $\alpha$ (a), with maximum angle of fracture $\alpha$ under different connective probability $p$ (b); development of $A_{0}$ with connective probability $p$ under different maximum angle of fracture $\alpha$ (c), with maximum angle of fracture $\alpha$ under different connective probability $p(\mathrm{~d})$.

\section{ACKNOWLEDGMENT}

This research is funded by the National Key Basic Research Program of China "Nos. 2006CB705800; 2011CB707302”.

\section{REFERENCES}

1. Bachu, S., $\mathrm{CO}_{2}$ storage in geological media: role, means, status, and barriers to deployment, Prog. Energ. Combust., 2008, vol. 34, pp. 254-273.

2. Jan, M.N., Dmitri, K., Celia, M.A., and Bachu, S., Model for $\mathrm{CO}_{2}$ leakage including multiple geological layers and multiple leaky wells, Environ. Sci. Technol., 2009, vol. 43, pp. 743-749.

3. Prasad, S., James, A., Fred, R., and Neeraj, G., Design of sensor networks for long term monitoring of geological sequestration, Energ. Convers. Management, 2006, vol. 47, pp. 1968-1974.

4. Alkan, H., Percolation model for dilatancy-induced permeability of the excavation damaged zone in rock salt, Int. J. Rock Mech. Mining Sci., 2009, vol. 46, no. 4, pp. 716-724.

5. Robinson, P.C., Connectivity of fracture systems: a percolation theory approach, J. Phys. A: Math. Gen., 1983, vol. 16, pp. 605-614.
6. Warren, J.E. and Root, P.J., The behavior of naturally fractured reservoirs, SPE J., 1963, vol. 3, no. 3, pp. 245-255.

7. Bunde, A. and Havlin, S., Fractals and Disordered System, Berlin: Springer-Verlag, 1996.

8. Stauffer, D. and Aharony, A., Introduction to Percolation Theory, London: Taylor \& Francis, 1992.

9. Sahimi, M., Appl. Percolation Theory, London: Taylor \& Francis, 1993.

10. Mourzenko, V.V., Thovert, J.F., and Adler, P.M., Macroscopic permeability of three-dimensional fracture networks with power-law size distribution, Phys. Rev. E., 2004, vol. 69, p. 066307.

11. Masihi, M., King, P.R., and Narafza, P., Fast estimation of connectivity in fractured reservoirs using percolation theory, SPE J., 2007, vol. 6, pp. 167-178.

12. Adler, P.M. and Thovert, J.F., Fractures and Fracture Networks, Dordrecht, Netherlands: Kluwer Academic, 1999.

13. Bonnet, E.O., Bour, N., Odling, P., et al., Scaling of fracture systems in geological media, Rev. Geophys., 2001, vol. 39, pp. 347-383.

14. Berkowitz, B., Characterizing flow and transport in fractured geological media: a review, Adv. Water Res., 2002, vol. 25, pp. 861-884. 
15. Hamzehpour, H., Mourzenko,V.V., Thovert, J.F., and Adler, P.M., Percolation and permeability of networks of heterogeneous fractures, Phys. Rev. E., 2009, vol. 79, p. 036302.

16. Sahimi, M., Flow and Transport in Porous Media and Fractured Rock, N.Y.: VCH, 1995.

17. Havlin, S. and Ben-Avraham, D., Diffusion in disordered media, Adv. Phys., 1987, vol. 36, pp. 695-698.

18. Levitz, P., From Knudsen diffusion to Levy walks, Europhys. Lett., 1997, vol. 39, no. 6, pp. 593-598.

19. Orbach, R., Dynamics of fractal networks, Science, 1986, vol. 231, pp. 814-819.

20. Klammler, F. and Kimmich, R., Geometrical restrictions of incoherent transport of water by diffusion in protein of silica fine particle systems and by flow in a sponge - a study of anomalous properties using an NMR field-gradient technique, Croatica Chemica Acta, 1992, vol. 65, no. 2, pp. 455-470.

21. Kimmich, R., Klammler, F., Skirda, V.D., et al., Geometrical restrictions of water diffusion in aqueous protein systems. A study using NMR Field-gradient techniques, Appl. Magn. Reson., 1993, vol. 4, pp. 425-440.
22. Kimmich, R., Stapf, S., Seitter, R.O., et al., Molecular dynamics of monomer, oligomer, and polymer liquids in porous media, a field cycling NMR relaxometry and NMR field-gradient diffusometry study, in Dynamics in Small Confining Sytems, Dyake, J.M., et al., Eds., Proc. Mater. Res. Soc. Symp. Proc. Mat. Res. Soc., Pittsburgh, 1995, vol. 189, pp. 178-200.

23. Kang, T.H., Zhao, Y.S., and Jin, Z.M., Fractal study of crack scale distribution in coalmass, J. China Coal Society, 1995, vol. 20, no. 4, pp. 393-398 [in Chinese].

24. Hestir, K. and Long, J.C.S., Analytical expressions for the permeability of random two-dimensional poisson fracture networks based on regular lattice percolation and equivalent media theories, J. Geophys. Res., 1990, vol. 95(B13), pp. 565-581.

25. Balberg, I., Anderson, C.H., Alexander, S., et al., Excluded volume and its relation to the onset of percolation, Phys. Rev. B, 1984, vol. 30, no. 7, pp. 39333943.

26. Brian, B., Analysis of fracture network connectivity using percolation, Theor. Math. Geol., 1995, vol. 27, no. 4, pp. $467-483$. 